\title{
Monitoring hot exciton dissociation in hybrid lead halide perovskite films with sub-10 fs pulses
}

\author{
Tufan Ghosh ${ }^{1}$, Sigalit Aharon ${ }^{1}$, Lioz Etgar ${ }^{1}$, and Sanford Ruhman ${ }^{1, *}$ \\ ${ }^{1}$ Institute of Chemistry, The Hebrew University, Jerusalem 91904, Israel
}

\begin{abstract}
Sub-10 fs pump-probe experiments on methylammonium lead halide $\left(\mathrm{MAPbI}_{3}\right)$ perovskite films show hot exciton dissociation in $20 \mathrm{fs}$ after photo-excitation with $\sim 0.7 \mathrm{eV}$ excess energy compared to its optical band gap (BG). Coherent wave packets were also detected in the form of spectral modulation, revealing electron-phonon coupling in these materials. The estimated electron-phonon coupling strengths from the frequency and amplitude of the detected spectral modulation are in the weak regime, suggesting formation of large polaron.
\end{abstract}

\section{Introduction}

Understanding high power conversion efficiency of perovskite-based solar cells requires a broad knowledge of the following phenomena: dissociation of exciton to free carriers, hot carrier cooling and carrier recombination dynamics [1,2]. Under solar irradiation, most of the photons absorbed in $\mathrm{MAPbI}_{3}$ possess $\sim 0.4 \mathrm{eV}$ of excess energy above its $1.65 \mathrm{eV} \mathrm{BG}$, and thus characterizing the important stages of free carrier generation and relaxation dynamics is essential. Carrier cooling and recombination in hybrid perovskites occurs in ps and ns timescales, respectively. Probing exciton dissociation, however, requires extreme (sub-10 fs) time resolution. The significance of optic phonon coupling to free carriers in these materials (in the form of polarons) has recently been a subject of lively debate [3]. To date, only a few time domain measurements have reported impulsive phonon activity, and those reports have not converged in terms of the active phonon frequencies or coupling strengths.

Here, we present high $\mathrm{S} / \mathrm{N}$ sub-10 fs pump-probe spectroscopy of $\mathrm{MAPbI}_{3}$ films to record ultrafast exciton dissociation, which has not been reported previously. Results from above band gap excitation show that instantly generated localized hot excitons dissociate to free carriers within $\sim 20$ fs after photoexcitation. Faint spectral modulations in the transient signals have been observed and assigned to coherent phonons. These modulations are assigned to longitudinal optical (LO) phonons weakly coupled to the electronic transition.

*Corresponding author: sandy@mail.huji.ac.il 


\section{Results and Discussion}

High above band gap excitation in $\mathrm{MAPbI}_{3}$ provides an opportunity to observe free carrier generation followed by cooling dynamics. The absorption spectra of the $\mathrm{MAPbI}_{3}$ films and pump pulse are shown in Fig. 1a. Transient transmission (TT) spectra of $\mathrm{MAPbI}_{3}$
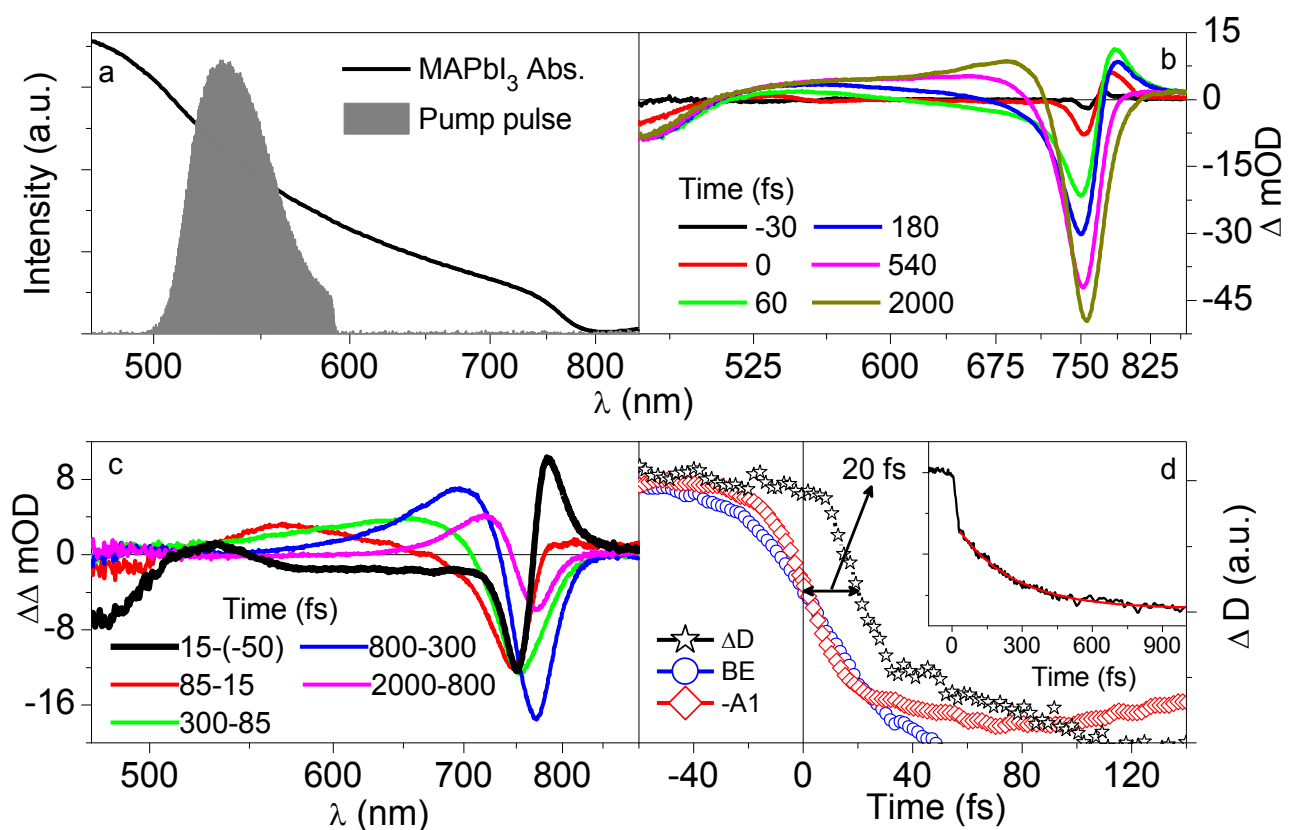

Fig. 1. (a) Absorption spectrum of $\mathrm{MAPbI}_{3}$ films and pump spectrum. (b) Transient difference transmission and (c) finite different spectra for $\mathrm{MAPbI}_{3}$ films after $530 \mathrm{~nm}$ excitation. Comparison of temporal evolution between band edge (BE) bleach, below BE photoinduced absorption (A1) and band integral $(\Delta \mathrm{D})$ (calculated over 550-900 $\mathrm{nm}$ range). Inset shows band integral up to $2 \mathrm{ps}$.

films after photoexcitation (Fig. 1b) reveal a number of features that are common to previous pump-probe studies of this system [2]. First, an intense photoinduced bleach at the optical BG, which appears instantly and grows to its full magnitude during carrier cooling (Fig. 2a), is attributed to hot carrier induced screening of the exciton transition and state filling. Another is a photoinduced absorption (PIA) feature rising below the band edge, assigned to BG renormalization and shifting of the exciton transition. This feature also appears immediately after excitation, but decays over the carrier cooling stage (Fig. 2b). The third is a slow rising broad PIA feature in the inter-band region.

Fig. 1c presents finite difference spectra showing evolution of probe transmission over a limited range of pump-probe delay. At early time (15-(-50) fs or black line in Fig. 1c) a sharp red shift of exciton transition is observed. At later times the spectral changes show a dominant bleaching due to state filling of the cooling carriers, peaked at the BG. A plot of a band integral $(\Delta \mathrm{D})$ of TT spectra over the range from $550-900 \mathrm{~nm}$ is presented in Fig. $1 \mathrm{~d}$. Surprisingly, the photoinduced band bleach (negative jump in $\Delta \mathrm{D}$ ) appears abruptly with a delay of $20 \mathrm{fs}$ after the pump excitation (Fig. 1d). This delay in band integral is assigned to exciton dissociation or the breakup of localized e-h pairs, characterized by the black spectrum in Fig. 1c. To the best of our knowledge, this is the first recording of this phase of free carrier formation following above BG photoexcitation in this material, or in bulk semiconductors. Observing this phase hinges on the ultrashort pump pulses ability to generate such localized carrier pairs even high above the BG due to coherent excitation of a 
broad band of "k" states. Finally, the delayed $\sim 0.4$ ps rise of the band integral is compatible with carrier cooling.
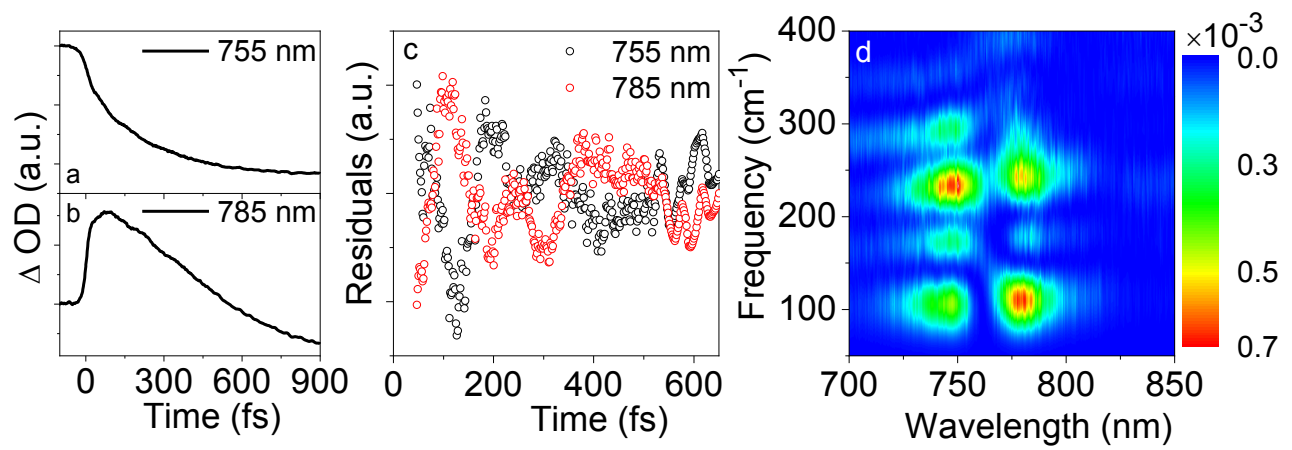

Fig. 2. (a, b) Temporal evolutions, and (c) Residual modulations at 755 and $785 \mathrm{~nm}$ for $\mathrm{MAPbI}_{3}$ films. (d) Two dimensional map of Fourier power for $\mathrm{MAPbI}_{3}$ films. The $x$-axis presents dispersed probe wavelength while the $y$-axis denotes Fourier frequency. The Fourier power is depicted by color coding according to the ruler show in the right.

The pump-probe spectra of $\mathrm{MAPbI}_{3}$ also reveal weak periodic modulations, due to coupling of the free carriers to the optic phonons. The residual modulations at different probe wavelengths were extracted by subtracting the transient signals (Fig. 2c). The Fourier analysis of the residual modulations detect predominantly two of active phonon modes: low frequency mode $\sim 110 \mathrm{~cm}^{-1}$, assigned to the $\mathrm{Pb}-\mathrm{I}$ stretching and high frequency mode $\sim 240$ $\mathrm{cm}^{-1}$, assigned to the torsions of methylammonium cation (Fig. 2d). The frequency and amplitude of these spectral modulations were used to estimate the electron-phonon coupling strength, assuming a displaced harmonic oscillator model. The estimated coupling strengths (Huang-Rhys parameter, S: $0.02-0.04$ ) for the $\mathrm{MAPbI}_{3}$ films are well within the weak coupling regime, which is compatible with free carriers existing as large polarons in these materials [4]. These observed phonon modes might not be responsible for perovskite's moderate carrier mobility, which requires intermediate electron-phonon coupling [5].

\section{Conclusions}

Sub-10 fs pump-probe spectroscopy of $\mathrm{MAPbI}_{3}$ films reveal an early stage of evolution where localized electron -hole pairs, exist even after above band gap excitation, and then dissociate to free carriers. We also detected impulsively excited coherent LO phonons which represent structural deformation in lead halide perovskite in fs time scale. Our experimental results estimate a weak coupling of these phonons to the electronic transition, suggesting formation of large polarons in these materials. These must therefore not be the degrees of freedom which dictate the intermediate carrier mobilities in this material [5].

\section{References}

1. J. Huang, Y. Yuan, Y. Shao and Y. Yan, Nat. Rev. Mater., 2, 17042 (2017).

2. V. Sharma, S. Aharon, I. Gdor, C. Yang, L. Etgar, S. Ruhman, J. Mater. Chem. A, 4, 3546 (2016).

3. K. Miyata et. al., Nat. Commun., 9, 1971 (2018).

4. T. Ghosh, S. Aharon, L. Etgar and S. Ruhman, J. Am. Chem. Soc., 139, 18262 (2017).

5. M. Sendner et al., Mater. Horiz. 3, 613 (2016). 